Military Technical College Kobry El-Kobbah, Cairo, Egypt

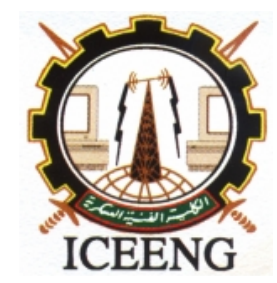

\author{
$7^{\text {th }}$ International Conference \\ on Electrical Engineering \\ ICEENG 2010
}

\title{
Application of Blind Adaptive LMS Algorithm for Detection of CDMA Signals in Frequency Selective Fading Channels
}

By

Salah. S. Elagooz**

Atif. L. Salama**

Eissa D Eissa *

\section{$\underline{\text { Abstract: }}$}

The conventional matched filter (MF) receiver is considered the optimum filter to recover the CDMA signals. One of its problems is that its performance is significantly degraded due to the channel impairments and the increase of the multiple access interference (MAI). Parallel interference cancellation (PIC) is considered a simple yet effective multi-user detector for direct-sequence code-division multiple-access (DSCDMA) systems. However, its performance may deteriorate due to unreliable interference cancellation in the early stages. Thus, a partial PIC detector, in which partial cancellation factors (PCFs) are introduced to control the interference cancellation level, it has been developed as a remedy. Recently, an interesting adaptive multistage PIC algorithm was proposed. In this paper, an application of the least mean square (LMS) adaptive algorithm is presented by training the adaptive coefficients blindly to optimize the values of the PCFs. The performance of the presented receiver is measured in terms of bit error rate (BER) and compared with other receivers over frequency selective fading channel. It is found that the performance of the adaptive PPIC receiver is better than the performance of the other receivers in the frequency selective fading channel especially in the last stages.

\section{Keywords:}

Code division multiple access (CDMA), adaptive partial parallel interference cancellation (APPIC), least mean square (LMS), matched filter (MF)

\footnotetext{
** Egyptian Armed Forces

* Libyan Armed Forces
} 


\section{Introduction:}

Multi-user detection (MUD) is a technique for improving the performance of codedivision multiple-access (CDMA) systems. Different from the conventional single-user approach, MUD does not treat interference from other users as noise. The development of MUD algorithms can be dated back to the seminal work of Verdu. He proposed a multi-user receiver utilizing the maximum-likelihood criterion [1] and showed a great performance enhancement. However, he also showed that the computational complexity grows exponentially with the user number. The high computational complexity adversely affects its real-world applications. Thus, a variety of low-complexity suboptimum receivers were then proposed [2-4].

The subtractive type interference cancellation is known to be a simple and effective MUD algorithm. This type of MUD involves only vector operations making it a good candidate for real-world implementation. For a particular desired user, the subtractivetype canceller estimates interference from other users, regenerates, and cancels it from the received signal. This canceller is usually implemented with a multistage structure, and the temporary data decision for a stage is obtained from its previous stage.

The successive interference cancellation (SIC) cancels interference from other users one by one [5-7], while the parallel interference cancellation (PIC) cancels it all at one time [8-10]. To have the best performance, signal power ranking is necessary for SIC. Stronger signals usually have lower probability of decision errors and cancellation of these signals gives more significant result. For these reasons, SIC has better performance where users have unbalanced powers. However, SIC requires additional complexity for power ranking and its processing delay are also larger. By contrast, PIC cancels the interference disregarding to the interference power distribution and is more suitable for power balanced systems.

One problem associated with the PIC approach is that interference estimation may not be reliable in early stages. In other words, interference cancellation does not necessarily reduce interference. To alleviate this problem, partial PIC was then developed. Partial cancellation factors (PCFs) ranging from zero to unity were introduced to control the signal cancellation level. Optimal PCFs can be theoretically derived with given channel and noise statistics. One problem of the approach is that the computational complexity will become very high in time-varying environments. Also, when the required statistics are not properly estimated, the performance may be seriously affected. To remedy the problem, an adaptive approach using the least mean square (LMS) algorithm was then 
proposed for partial PICs [11]. Due to the special architecture proposed in [11], the adaptive algorithm does not require any training sequence. In other words, it is operated in a blind way. The basic idea behind the adaptive multistage PIC is fundamentally different from that of conventional partial PICs. The weight vector it derives for a user corresponds to a combination of the channel response and the PCF. There are many advantages using the adaptive PIC in [11]. It is simple to implement [12], and is inherently applicable in time-varying environments. Also, it does not have to conduct channel estimation, and its performance is better than non adaptive PICs. Other related works can be found in [13-16]. In [14], the adaptive multistage PIC was applied to multi-rate systems. In $[15,16]$, adapted weights are filtered before or after weight adaptation such that better cancellation performance can be obtained.

Although the adaptive multistage PIC has been studied by many researches, its performance has not been analyzed before. The difficulty arises from the nonlinear operation involved in the decision process, and its interaction with the LMS algorithm. There exist many theoretical results for the LMS algorithm; however, most of them consider the steady state performance and are valid only for the small step size scenario. This cannot be applied in the problem considered here. This is because in an adaptive PIC, only the data in one bit interval are available. For better performance, a large step size must be used. Even with the large step size, the weights still cannot converge due to the short training sequence. As a result, the LMS algorithm is always in its transient state. One other obstacle is that the input to the LMS algorithm in a certain stage depends on the decision in the previous stage, and this complicates the problem further more. In [17], a method overcoming these problems outlined above. it explicitly derives the analytical results for optimal weights, weight-error means, and weight-error variances. Based on these results, it finally derive the output bit error rate (BER) for each user. Simulation results indicate that the analytical results highly agree with empirical ones. Although the analysis provides only the system behavior of the original structure in [11], it is straightforward to conduct the performance analysis for the improved structures such as [15] or [16] with the proposed algorithm.

The remainder of the paper is organized as follows. In Section 2, the framework of the adaptive multistage PIC is first reviewed. In Section 3, numerical results are presented showing the performance of the adaptive PPIC receiver and compared with the traditional PPIC and PIC receivers in addition to the MF under the same conditions. Finally the conclusions are drawn in Section 3. 


\section{System Model:}

Consider a synchronous $K$ user CDMA system in the additive white Gaussian noise (AWGN) channel. Let the spreading sequence of the $K^{\text {th }}$ user denoted by $x_{k}(n)$ with processing gain $N$ and amplitude $\pm 1 / \sqrt{N}$. Then the chip-sampled received signal in a certain bit interval can be represented as

$r(n)=\sum_{k=1}^{N} a_{k} b_{k} x_{k}(n)+v(n)$

$n=0, \ldots . ., N-1$

where $a_{k}$ and $b_{k}$ are the channel gain and data bit of the $K^{\text {th }}$ user, and $v(n)$ is the AWGN with variance $\sigma^{2}$. Without loss of generality, we let $a_{k}>0$. We first describe the operation of a partial PIC receiver. The first stage operation is just the matched filtering. Let $y_{k}^{(i)}$ be the $i^{\text {th }}$ stage output of the $K^{\text {th }}$ user. Then,

$\mathrm{y}_{\mathrm{k}}^{(1)}=\sum_{\mathrm{n}=0}^{\mathrm{N}-1} \mathrm{x}_{\mathrm{k}}(\mathrm{n}) \mathrm{r}(\mathrm{n})=\mathrm{a}_{\mathrm{k}} \mathrm{b}_{\mathrm{k}}+\sum_{\mathrm{j} \neq \mathrm{k}} \mathrm{a}_{\mathrm{j}} \mathrm{b}_{\mathrm{j}} \rho_{\mathrm{jk}}+\sum_{\mathrm{n}=0}^{\mathrm{N}-1} v_{\mathrm{k}}(\mathrm{n})$

where $\rho_{j k}=\sum_{n=0}^{N-1} x_{j}(n) x_{k}(n)$ defined as the time averaged cross-correlation function between the $i^{\text {th }}$ and the $j^{\text {th }}$ user and $v_{k}(n)=x_{k}(n) v(n)$. We further denote the noise term in (2) as $\gamma_{k}=\sum_{n=0}^{N-1} v_{k}(n)$. It can be seen that in the received signal of (2), in addition to noise, the MAI affects the signal detection. Let $\hat{r}_{k}^{(i)}(n)$ denote an interference-subtracted signal for User $k$ in the $i^{\text {th }}$ stage and $i>1$. Then,

$$
\begin{aligned}
\hat{r}_{k}^{(i)} & =r(n)-\sum_{j \neq k} g_{j}^{(i)} \hat{s}_{j}^{(i)}(n) \\
& =r(n)-\sum_{j \neq k} g_{j}^{(i)} a_{j} \hat{b}_{j}^{(i-1)} \cdot x_{j}(n)
\end{aligned}
$$

where $g_{j}^{(i)}$ denotes the PCF for the $j^{\text {th }}$ user in the $i^{\text {th }}$ stage and $\hat{s}_{j}^{(i)}(n)$ is the corresponding interference estimate being equal to $a_{j} \hat{b}_{j}^{(i-1)} \cdot x_{j}(n)$. Thus, the $j$ output signal in Stage $i$ is then

$$
y_{k}^{(i)}=\sum_{n=0}^{N-1} \hat{r}_{k}^{(i)}(n) x_{k}(n)
$$

Finally, we can obtain the $j^{\text {th }}$ stage detected bit with $y_{k}^{(i)}$, i.e., $\hat{b}_{j}^{(i)}=\operatorname{sgn}\left[y_{k}^{(i)}\right]$ in which sgn[.] denotes the sign operation. As we can see, the knowledge of the channel gain is required in partial PIC. In general, optimal PCFs are derived through minimization of some cost functions. The interference subtraction operation of the adaptive multistage PIC is similar to that of the conventional partial PIC. However, the PCF and the channel gain are merged into a single weight. Let $w_{k}^{(i)}$ be the weight of the $K^{\text {th }}$ user at the $j^{\text {th }}$ stage 
and $\mathbf{w}^{(i)}=\left[w_{1}^{(i)}, w_{2}^{(i)}, \ldots, w_{K}^{(i)}\right]^{T}$. The optimal weight vector, denoted as $\mathbf{w}_{\mathrm{opt}}^{(i)}$, is obtained by minimizing a mean square error (MSE) function $j^{(i)}$, i.e. $\mathbf{w}_{\mathrm{opt}}^{(i)}=\min _{\mathbf{w}^{(i)}} j^{(i)}$. The MSE is given $j^{(i)}=E\left\{\left[r(n)-\widetilde{r}^{(i)}(n)\right]^{2}\right\}$.

$j^{(i)}=E\left\{\left[r(n)-\tilde{r}^{(i)}(n)\right]^{2}\right\}$

$$
=E\left\{\left[r(n)-\sum_{k=1}^{K} w_{k}^{(i)} \hat{b}_{k}^{(i-1)} x(n)\right]^{2}\right\}
$$

Note that $\tilde{r}^{(i)}(n)$ denotes the reconstructed received signal (excluding noise). The optimum solution of (5) can be theoretically solved. However, it will require high computational complexity. A simple alternative is to use the adaptive filtering approach. The LMS algorithm is a well-known adaptive algorithm. Let $x(n)=\left[x_{1}(n), x_{2}(n), \ldots \ldots, x_{K}(n)\right]^{T}$ The LMS update equation for the $i^{\text {th }}$ stage processing (with $i-1$ stages of interference cancellation) can be expressed as

$\hat{r}^{(i)}(n)=\left[\chi^{(i)}(n)\right]^{T} \mathbf{w}^{(i)}(n)$

$e^{(i)}(n)=r(n)-\hat{r}^{(i)}(n)$

$\mathbf{w}^{(i)}(n+1)=\mathbf{w}^{(i)}(n)+\mu e^{(i)}(n) \chi^{(i)}(n)$

where the input signals are described as $\chi^{(i)}(n)=\hat{B}^{(i-1)} x(n)$ with $\hat{B}^{(i)}=\operatorname{diag}\left\{\hat{b}_{1}^{(i)}, \hat{b}_{2}^{(i)}, \ldots \ldots ., \hat{b}_{K}^{(i)}\right\}$ After the weights are trained for a bit interval, they are used to cancel the interference from other users such that the input to the $K^{\text {th }}$ user' slicer in the $i^{\text {th }}$ stage is

$\hat{r}_{k}^{(i)}=r(n)-\sum_{j \neq k} w_{j}^{(i)}(N) \hat{b}_{j}^{(i-1)} \cdot x_{j}(n)$

Then the $i^{\text {th }}$ stage output for the $K^{\text {th }}$ user can be obtained as that in (4). The block diagrams for the adaptive multistage partial PIC and the LMS algorithm are shown in Figs. 1 and 2, respectively. In this paper, we will consider an adaptive two-stage PIC receiver. Since only the operation in the second stage is concerned, the superscripts for the first-stage outputs and for the second-stage inputs are omitted for notational simplicity. As a result $y_{k}=y_{k}^{(1)}, b_{k}=\hat{b}_{k}^{(1)}, \widetilde{r}(n)=\widetilde{r}^{(2)}(n)$ and $\widetilde{w}(n)=\widetilde{w}^{(2)}(n)$. It can be seen from (5) that in the ideal condition, i.e., $\widetilde{r}(n)$ is equal to the noise-free received signal, the ideal convergent weights will be

$$
w_{k}(N)= \begin{cases}a_{k}, & \hat{b}_{k}=b_{k}, \\ -a_{k}, & \hat{b}_{k} \neq b_{k} .\end{cases}
$$




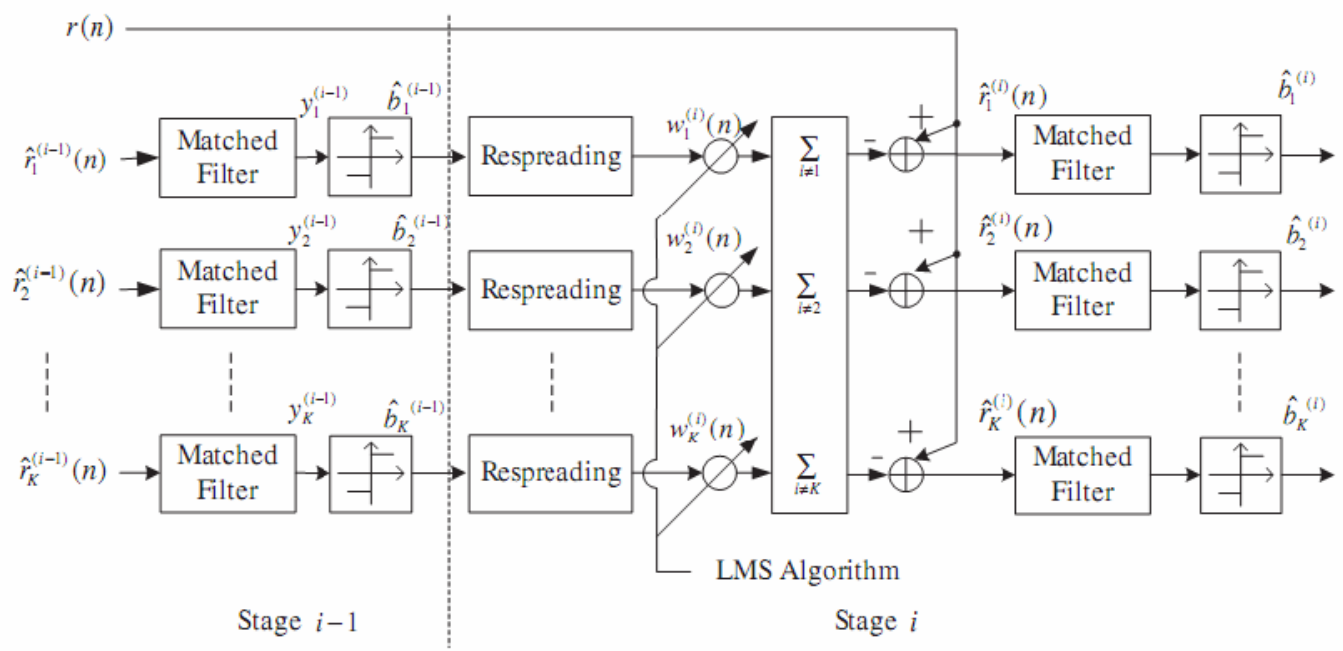

Figure (1): Block diagram of an adaptive multistage PIC receiver.

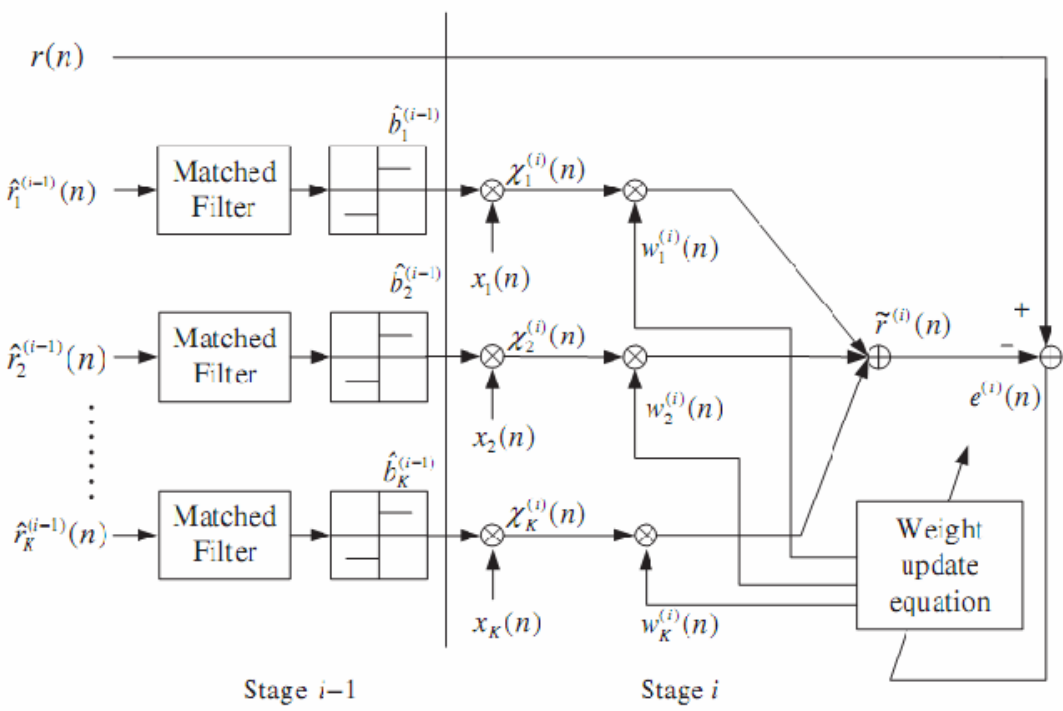

Figure (2): LMS algorithm for an adaptive multistage PIC receiver.

Thus, the convergent weights depend on whether the bit decisions in the previous stage are correct or erroneous. This property makes the performance analysis difficult. The adaptive algorithm allows the weight of each user to attain the desired value bit by bit. This is the reason why the adaptive approach performs better than non-adaptive methods.

As mentioned, the adaptation period is constrained in one symbol period. This is 
because the optimal weight for User $k$ may be $+a_{k}$ or $-a_{k}$ depending on the bit decision for each symbol. Although the LMS algorithm is simple, its convergence is slow and the weight may not converge to the desired value in such a short period. In addition, the resultant weight heavily depends on the parameters used in the LMS algorithm so is the cancellation performance. These parameters include the step size and initial weights. In the conventional approach, these parameters are determined heuristically. The weight initials are usually set as the channel gains, i.e. $w_{k}(0)=a_{k}$. This is reasonable since the bit error probability is usually low, most of the weights will start adaptation at their optimal values; only few weights are away from their optimal values by $2 a_{k}$. A larger step size will accelerate the convergence speed for the weights with erroneous decision, but also inevitably introduces a larger variance for all weights. There is almost no research regarding the convergence analysis for the adaptive multistage PIC receiver.

\section{Simulation Results:}

In this section, the performance of the adaptive partial PIC receiver is evaluated and compared with the performance of the traditional partial PIC and the conventional PIC receivers. Moreover, the performances of the adaptive partial PIC receiver is compared with the performance of the matched filter of a single user (SUMF) in AWGN channel, which is considered as a reference for the other receivers. The comparison also includes the performance of the matched filter in presence of the same number of MAI under the same conditions in frequency selective fading channel.

All the simulations are performed under nearly identical conditions to make the comparison fair as much as possible. The simulations are performed using 5000 random transmitted symbol for each user and averaged over 250 independent trials to make the complexity of the program visible. The Gold code with code length 15 is used as spreading code. We use a Gold code in the simulation because it has the availability to generate a large number of sequences with good cross correlations. The convergence simulations are taken over the period of the first bite for the LMS algorithm since the adaptation process is performed blindly. The step size parameter is adjusted according to the other simulation parameters (i.e. the number of MAI, the code length... etc).

Fig (3) represents the BER against the SNR. The figure compares the performance of the adaptive partial PIC receiver, traditional partial PIC, and conventional PIC receivers with the performance of the SUMF, and the MF under the effect of $5 \mathrm{MAI}$ in frequency selective fading channel. The reason of including the SUMF is that its performance is considered as the lower bound (a reference performance) of the performances of the 
other CDMA receivers, since it represents the performance of the MF in AWGN channel without any effect of interfering users. The figure shows that the performance of the MF represent the worst performance due to the effect of the MPI, while the PIC receivers generally introduce a superior performance compared with the MF. The reason of the large gape between the PIC receivers and the MF receiver is the deterioration effect of the MPI on the MF while it has very little effect on the PIC receivers. More over, the low number of interfering users decreases also the effect on the PIC receivers. For the comparison between the PIC receivers, it is clear that the two stages of the traditional PPIC are better than the two stages of the conventional PIC. For the proposed APPIC it is found that its first stage introduce an enhancement by about $1 \mathrm{~dB}$ over the first stage of the conventional PIC, and about $0.5 \mathrm{~dB}$ over the traditional PPIC at BER of $10^{-4}$. It also found that the second stage of the APPIC completely coincident with the SUMF, which mean that it has the same performance of the SUMF. That gives an indication that the proposed APPIC completely eliminate all the effects of the MPI and all the 5 interfering users. The reason of this superior performance is due to using optimized PCFs which optimized the fraction of the MAI cancellation in the tow stages of the proposed APPIC receiver. Of course, the PCFs are optimized through out the adaptation process.

Fig (4) illustrates the same performance comparison of the two stages PIC receivers and the MF but when the number of interfering users is increased to 9 MAI. The figure shows that the performance of the MF is still completely deteriorated all over the SNR range. The figure shows also that at low SNR, the performances of all the PIC receives stages are closed to each other; the reason of this phenomenon is that the effect of the noise is still dominant. As the SNR increased to be over $6 \mathrm{~dB}$, the performances of the PIC receivers begin to be contrasted. The figure shows that increasing the MAI affects on the performance of the first stages of the conventional PIC and the traditional PPIC receives, and even the proposed APPIC. That can be cleared from the gape between the first stages of the PIC receivers and the SUMF, which ranging between 2 and $3 \mathrm{~dB}$ at BER $10^{-4}$. For the case of the second stages, it is clear that increasing the MAI affects only on the PIC and the traditional PPIC receives, while for the proposed APPIC receiver, the second stage still coincident completely with the SUMF, which mean that although the MAI interference increased, the proposed APPIC receiver still eliminates its effect over the frequency selective fading channel.

Fig (5) illustrates the performance comparison in case of the full system capacity. This means that the maximum number of interfering users is applied. In case of using the Gold code with length 15, the maximum available interfering users are 14 in addition to the desired user. The figure shows that the first stages of all the PIC receiver types are 
strongly affected by the increased number of MAI. For the second stages, the figure shows that conventional PIC is strongly affected by the highly increased number of MAI; meanwhile the traditional PPIC still can resist the increasing of the MAI with performance gain $2.8 \mathrm{~dB}$ with the second stage of the conventional PIC at BER $10^{-4}$. The second stage of the proposed APPIC presents the best performance compared with the second stages of the traditional PPIC and conventional PIC; it presents a performance enhancement $3.2 \mathrm{~dB}$ with the second stage of the conventional PIC, and 1 $\mathrm{dB}$ with the second stage of the traditional PPIC at BER $10^{-4}$.

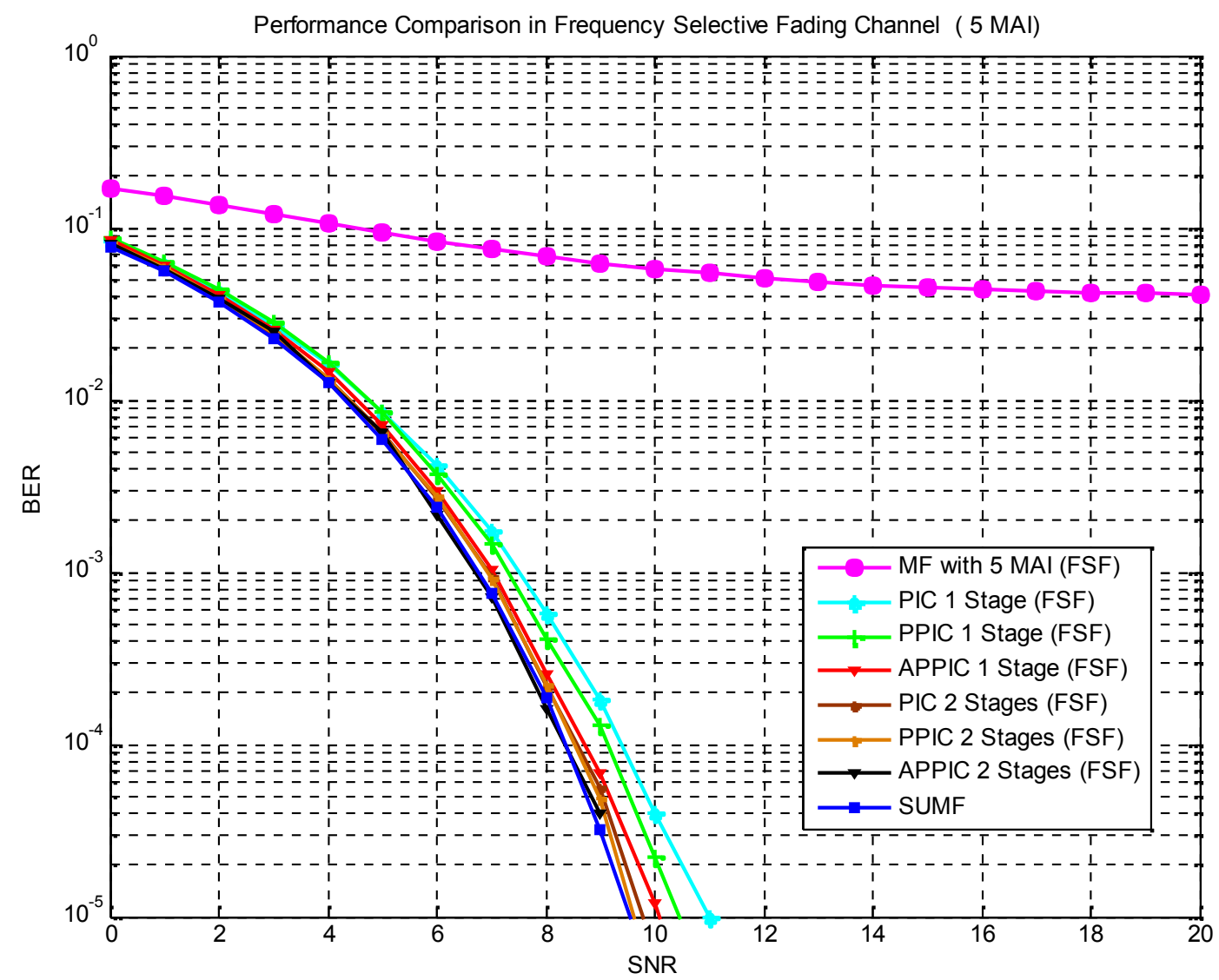

Figure (3): Performance Comparison of the APPIC, PPIC, PIC, and MF (5 MAI) in Frequency Selective Fading Channel 


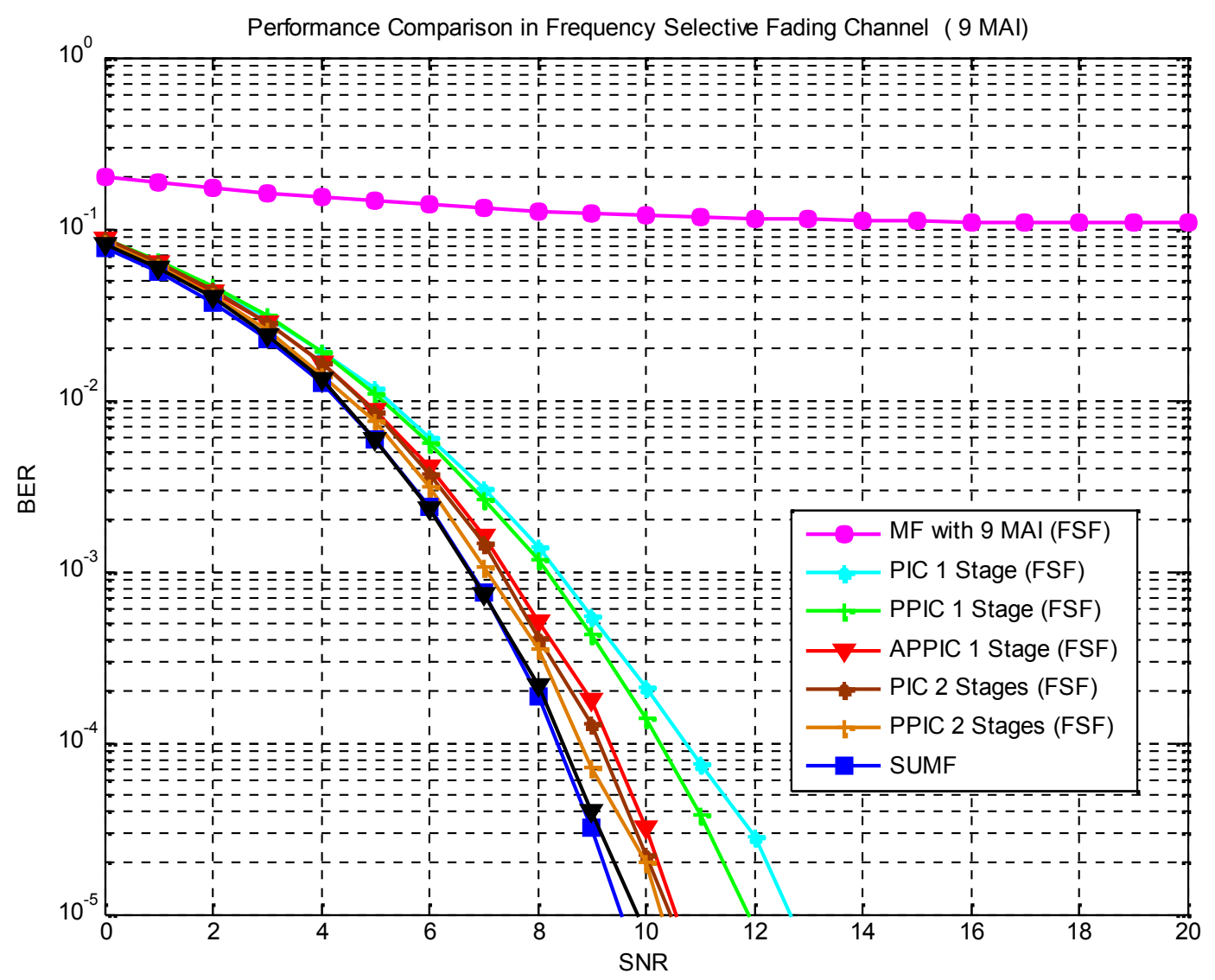

Figure (4): Performance Comparison of the APPIC, PPIC, PIC, and MF (9 MAI) in Frequency Selective Fading Channel 


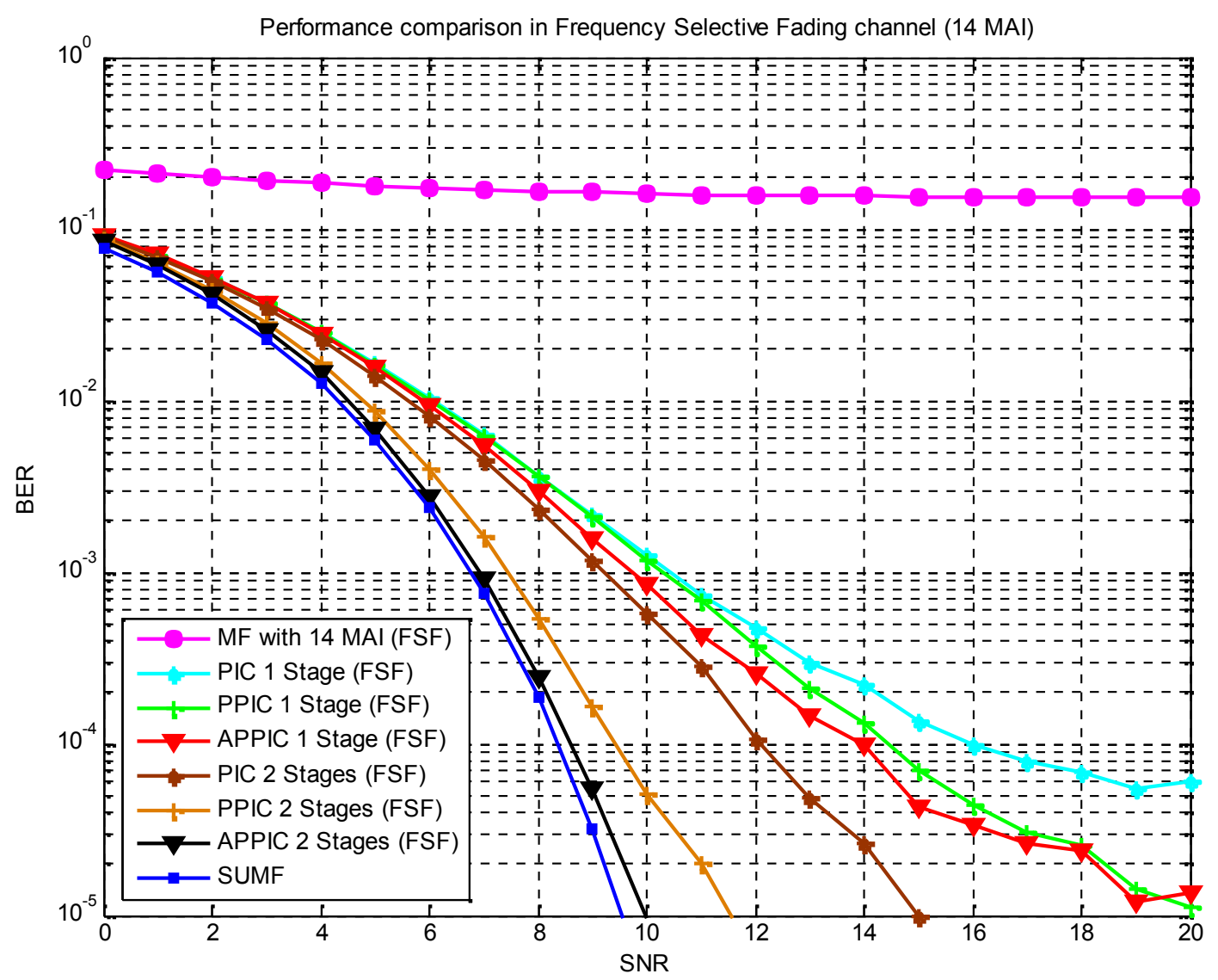

Figure (5): Performance Comparison of the APPIC, PPIC, PIC, and MF (14 MAI) in Frequency Selective Fading Channel

\section{Conclusions:}

Parallel interference cancellation (PIC) is considered a simple yet effective multi-user detector for direct-sequence code-division multiple-access (DS-CDMA) systems. However, its performance may deteriorate due to unreliable interference cancellation in the early stages. Thus, a partial PIC detector, in which partial cancellation factors (PCFs) are introduced to control the interference cancellation level, has been developed as a remedy. The PCFs can be chosen such that it does not give the best performance, for this reason an adaptive PPIC receiver is presented, in which the PCFs are optimized through out adaptation process. The adaptation process using the least mean square (LMS) algorithm as an adaptation algorithm. A performance comparison performed between the conventional PIC, traditional PPIC, and the proposed APPIC receiver, in addition to the MF under the same condition and the SUMF is taken as a reference. It is found that 
in low number of MAI, all the PIC receivers introduce superior performances for the two stages over the MF in the frequency selective fading channel. It is found also that proposed APPIC receiver completely eliminates the effect of the MAI, As the MAI increased, the first stages of the PIC recovers are strongly affected, while the second stages still presents a very good performances, and the proposed APPIC receiver still completely eliminate the effect of the MAI, and the channel. As the MAI reaches to the maximum system capacity, the two stages of the conventional PIC receivers are strongly affected while the PPIC and the APPIC second stages are still having better performance.

\section{References:}

[1] S. Verdu, Minimum probability of error for asynchronous Gaussian multipleaccess channels, IEEE Trans. Inform. Theory IT-32 (January 1986) 85-96.

[2] S. Moshavi, Multi-user detection for DS-CDMA communications, IEEE Comm. Mag. 34 (10) (1996) 124-136.

[3] A. Duel-Hallen, J. Holtzman, Z. Zvonar, Multiuser detection for CDMA systems, IEEE Personal Comm. 2 (2) (1995) 46-58.

[4] S. Verdu, Multiuser Detection, Cambridge University Press, Cambridge, UK, 1998.

[5] A.J. Viterbi, Very low rate convolutional codes for maximum theoretical performance of spread-spectrum multiple-access channels, IEEE J. Select. Areas Comm.8 (4) (1990) 641-649.

[6] J.M. Holtzman. Successive interference cancellation for direct sequence code division multiple access. Military Conf., MILCOM '94, vol. 3, 1994, pp. 9971001.

[7] P. Patel, J.M. Holtzman, Analysis of simple successive interference cancellation scheme in DS/CDMA system, IEEE J. Select. Areas Comm. 12 (5) (1994) 796 807.

[8] M.K. Varanasi, B. Aazhang, Multistage detection in asynchronous code-division multiple-access communications, IEEE Trans. Comm. 38 (4) (1990) 509-519.

[9] R. Kohno, H. Imai, M. Hatori, S. Pasupathy, An adaptive canceller of cochannel interference for spread-spectrum multiple-access communication networks in a power line, IEEE J. Select. Areas. Comm. 8 (4) (1990) 691-699.

[10] Y.C. Yoon, R. Kohno, H. Imai, A spread-spectrum multiaccess system with cochannel interference cancellation,IEEE J. Select. Areas Comm. 11 (7) (1993) $1067-1075$.

[11] G. Xue, J. Weng, T. Le-Ngoc, S. Tahar, Adaptive multistage parallel interference 
cancellation for CDMA, IEEE J. Select. Areas Comm. 17 (10) (1999) 1815-1827.

[12] Y.B. Guo, J.R. Cavallaro, A low complexity and low power SoC design architecture for adaptive MAI suppression in CDMA systems, J. VLSI Signal Process. 44 (3) (2006) 195-217.

[13] S.R. Kim, I. Choi, S. Kang, J.G. Lee, Adaptive weighted parallel interference cancellation for CDMA systems, Electron. Lett. 34 (22) (1998) 2085-2086.

[14] S.H. Han, J.H. Lee, Adaptive multi-stage parallel interference cancellation receiver for multi-rate DS-CDMA system, IEICE Trans. Comm. E86-B (1) (2004) 2401-2405.

[15] Y.-F. Huang, J.-H. Wen, S.T. Wu, An adaptive decisionfeedback multiuser detector using parallel interference cancellation for CDMA systems, in: Proceedings of the IEEE VTC 2001 Spring, vol. 3, May 2001, pp. 1770-1774.

[16] J. Bae, I. Song, D.H. Won, A selective and adaptive interference cancellation scheme for code decision multiple access systems, Signal Processing 83 (2) (2003) 259-273.

[17] Yu-Tao Hsieh, Wen-Rong Wub, Performance analysis of an adaptive two-stage PIC CDMA receiver in AWGN channels, Signal Processing 88 (2008) 14131427. 